\title{
A bacterial secretosome for regulated envelope biogenesis and quality control
}

\author{
Daniel W. Watkins and Ian Collinson ${ }^{\dagger}$
}

\author{
School of Biochemistry, University of Bristol, BS8 1TD, United Kingdom
}

${ }^{\dagger}$, corresponding author: $\underline{\text { ian.collinson@bristol.ac.uk }}$

\section{SUMMARY}

As the first line of defence against antibiotics, the Gram-negative bacterial envelope and its biogenesis are of considerable interest to the microbiological and biomedical communities. All bacterial proteins are synthesised in the cytosol, so inner- and outer-membrane proteins, and periplasmic residents have to be transported to their final destinations via specialised protein machinery. The Sec translocon, a ubiquitous integral inner-membrane (IM) complex, is key to this process as the major gateway for protein transit from the cytosol to the cell envelope; this can be achieved during their translation, or afterwards. Proteins need to be directed to the innermembrane (usually co-translational), otherwise SecA utilises ATP and the proton-motive-force (PMF) to drive proteins across the membrane post-translationally. These proteins are then picked up by chaperones for folding in the periplasm or delivered to the $\beta$-barrel assembly machinery (BAM) for incorporation into the outer-membrane. The core heterotrimeric SecYEG-complex forms the hub for an extensive network of interactions that regulate protein delivery and quality control. Here, we conduct a biochemical exploration of this 'secretosome' - a very large, versatile and inter-changeable assembly with the Sec-translocon at its core; featuring interactions that facilitate secretion (SecDF), inner- and outer-membrane protein insertion (respectively, YidC and BAM), protein folding and quality control (e.g. PpiD, YfgM and $\mathrm{FtsH}$ ). We propose the dynamic interplay amongst these and other factors act to ensure efficient whole envelope biogenesis, regulated to accommodate the requirements of cell elongation and division. This organisation would be essential for cell wall biogenesis and remodelling and thus its perturbation would be a good strategy for the development of antimicrobials. 


\section{INTRODUCTION}

2

The bacterial envelope is essential for survival against an extraordinary range of physical and chemical environmental stresses. This protection has enabled their complete occupation of land and sea, including even the most inhospitable places and the invasion of more complex animal and plant hosts. While the envelope provides a barrier against the defence mechanisms deployed against bacteria, it is also an area of weakness for targeting by antibiotics and synthetic drugs.

The Gram-negative envelope is composed of a periplasm, containing a matrix of polymeric peptidoglycan (PG), sandwiched between inner- and outer-membranes. These membranes and intervening gelatinous space contain numerous proteins that need to be delivered, assembled and maintained at the right place (the inner- or outer-membrane, or periplasm), and time. Thus, the process is further complicated by the continuously changing state of the envelope, in response to changing environmental conditions and during cell division.

The classical view for this process involves the delivery of proteins to the inner-membrane - principally, the ubiquitous Sec machinery - for the delivery of proteins across or into it. Transport across the membrane, otherwise known as secretion, is usually achieved posttranslationally by the cytosolic ATPase SecA in conjunction with the inner-membrane proteinchannel complex SecYEG, facilitated by the ancillary sub-complex SecDFyajC [1] and driven by the proton motive force (PMF) [2-4]. By contrast, inner-membrane protein insertion occurs co-translationally, also via SecYEG, together with the highly conserved YidC $[5,6]$.

The SecYEG core-translocon, SecDFyajC and YidC combine to form a larger assembly known as the holo-translocon (HTL), capable of engaging in specialised post- and cotranslational translocation $[7,8]$, and providing protein substrates with alternative routes across or into the inner-membrane. Proteins destined for the periplasm, outer-membrane or beyond that are recognised by the HTL and must travel through the centre of SecYEG, while $\alpha$-helical membrane proteins slide through a lipid pool at the interface between SecYEG and YidC on their way to the bilayer of the inner-membrane [9].

Many of the proteins emerging into the periplasm are greeted by chaperones, such as SurA, Skp and DegP [10,11], to facilitate folding, or degradation if they become mis-folded [12]. One class of such proteins are the outer-membrane proteins (OMPs) that primarily adopt a $\beta$-barrel 
1 transmembrane domain. These are delivered across the periplasmic space to the $\beta$-barrel

2 assembly machinery (BAM) complex in the outer membrane for insertion and folding [13, 14].

3 Recently, we have shown that the HTL and BAM connect to form a trans-periplasmic inter-

4 connection of the inner- and outer-membranes [15]. This most likely transient interaction

5 provides a contiguous, unrestricted (e.g. by-passing the PG layer) pathway directly to BAM for

6 outer membrane proteins (OMPs). Presumably this co-operation enables the required passage

7 of very large quantities of OMPs, while protecting the transiting unfolded polypeptide from

8 proteolysis and aggregation, for high maturation efficiency. The assembly seems to be stabilised

9 by cardiolipin (CL) [15], a phospholipid required for the conferral of PMF stimulated secretion

10 of OMPs [16].

11 Considering the knowledge presented above, we reasoned that there may be a hitherto

12 unidentified trans-periplasmic complex, including the Sec and BAM complexes, plus several

13 other permanent and guest residents, responsible for envelope biogenesis, quality control and

14 cellular re-modelling. Such a complex may not have been distinguished before, e.g., by electron

15 microscopy because the envelope is very crowed, or biochemically because of its instability

16 and plasticity. The experiments described below explore the existence of such a secretosome

17 and some (but certainly not all) of its prospective constituents and clientele, as well as their

18 roles.

\section{RESULTS}

Very large membrane protein complexes of the $E$. coli envelope stabilised by cardiolipin

We began by analysing detergent extracts of total membranes of E. coli. Extracts were

24 produced by solubilising membranes with the detergent dodecyl-maltoside (DDM) and

25 subsequently fractionated by size exclusion chromatography. We know from previous findings

26 that cardiolipin (CL) is required to stabilise the associated states of SecYEG-SecDFyajC-YidC

27 (the holo-translocon) as well as the holo-translocon-BAM super-complex [17-19], so we

28 reasoned that if an even larger Sec-associated complexes existed in the envelope then they too

29 would be stabilised by CL. Thus, the size-exclusion experiments were conducted with and

30 without augmented CL in the column buffer $(0.02 \%(\mathrm{w} / \mathrm{v}) \mathrm{DDM} \pm 0.002 \%(\mathrm{w} / \mathrm{v}) \mathrm{CL})$.

31 Interestingly, inclusion of CL during the chromatography did indeed enable the preservation of 
1 entities with very high apparent molecular weights of approximately 460 - $1700 \mathrm{kDa}$ (calculated

2 using calibration standards shown in Fig. S1, top panel), represented by a peak at $8.9 \mathrm{~mL}$ (Fig.

3 1a, light blue trace), which were diminished without the lipid supplement to form a peak at 10.2

$4 \mathrm{~mL}(170$ - $460 \mathrm{kDa})$ (Fig. 1a, dark blue trace). The average size of the mixed assemblies

5 contained in both peaks were greater in size compared to purified SecYEG (Fig. 1a, magenta

6 trace, peak at $\sim 160 \mathrm{kDa}$, true molecular weight $=74 \mathrm{kDa}$ ), HTL (Fig. 1a, red trace, peak at $\sim 200$

$7 \mathrm{kDa}$, true molecular weight $=249 \mathrm{kDa})$ and BAM (Fig. 1a, orange trace, peak at $\sim 300 \mathrm{kDa}$, true

8 molecular weight $=209 \mathrm{kDa}$ ). Note the disparity between the apparent molecular weights of

9 SecYEG, HTL and BAM and their true molecular weights, due to their annular detergent

10 micelles and oligomerisation [20].

\section{A proportion of the envelope's Sec translocons form part of a much larger assembly}

13 The total E. coli membrane extract liberates a major early eluting peak, suggesting a high

14 proportion of the cell's total membrane proteins are contained within an assortment of large

15 membrane protein complexes, with many of them stabilised by CL. Given the Sec translocon

16 is formed of several proteins, and that it is known to interact with many others, we reasoned

17 that the Sec subunits would be found in the large molecular weight peak. Therefore, we took

18 the gel-filtrated fractions and looked for the individual proteins by immunoblotting. In the

19 absence of CL, SecY and SecG of the core translocon and SecD of the HTL form similar elution

20 profiles, mainly eluting between 10-12 mL (approximately 130 - $370 \mathrm{kDa}$, Fig. 1b-d, blue traces

21 vs red and magenta dashed lines), corresponding to the low molecular weight peak seen in the

22 chromatogram. In the presence of CL (Fig. 1b-d light blue trace), the elution profiles formed an

23 additional shoulder between 9 - $10 \mathrm{~mL}$ (approximately 370-790 kDa), suggesting the formation

24 of higher molecular mass complexes. In both the absence and presence of CL, the proteins elute

25 at a higher apparent molecular mass than their purified counterparts (Fig. 1b-d, blue traces vs

26 red and magenta dashed lines).

27 What other factors associate with SecYEG to account for this very large size difference? The 28 most obvious are the HTL components SecDFyajC and YidC [7, 21], confirmed here by the co29 elution of SecD with SecY and SecG (Fig. 1b-d). However, the HTL alone (Fig. 1b-d, red trace)

30 is not large enough to account for the early elution of the Sec subunits. Other factors must also 31 be associated. 
2 Known Sec interactors form CL-dependent high molecular weight complexes

Next, we analysed the size-exclusion fractions for known interactors of the Sec-machinery. Further Western blots confirmed the presence of BAM (BamA, BamB and BamD) in the higher

5 MW fractions, which was also dependent on CL (Fig. 1e-g), consistent with our previous findings [15]. The periplasmic chaperones YfgM and PpiD have both been shown to associate with one another and with the lateral gate of SecYEG for membrane protein departure [22, 23]. YfgM and PpiD were detected in the high MW peak along with the HTL and BAM (Fig. 1h, i). Once again, this associated state was preserved by CL. Finally, we looked for the inclusion of the $\mathrm{AAA}^{+}$ATP-dependent protease FtsH - a cytosolic inner-membrane anchored quality

11 control factor required for the degradation of misfolded proteins [24, 25] known to associate

12 with SecY and YidC [26-29]. Interestingly, the protein was detected in the same higher MW

13 fractions, but in this case independent of CL (Fig. 1j).

14 The overlapping, CL-dependent elution profiles of the Sec components and its known 15 interactors points towards the presence of large complexes in the E. coli membrane. The 16 existence of a 'secretosome' is a very intriguing prospect (discussed previously [15]). Thus, we

17 set out to understand more about its stability and additional interactors.

\section{Removal of CL from the secretosome results in dissociation of its components}

This secretosome is evidently prone to dissociation as its constituents were also detected in fractions corresponding to lower MWs (Fig. 1). This must be due, in part, to instabilities introduced by dissolution of the membranes and the extraction of specifically bound lipids -

23 partially ameliorated by the augmentation of CL in the extraction buffer. When the high MW

24 fractions were pooled and reapplied to the size exclusion column, the elution profiles of SecG,

25 SecD and BamA volume were highly dependent on CL (Fig. 2). In the presence of CL the 26 integrity of the higher MW complex containing the Sec and BAM complexes was maintained, 27 while its omission resulted in a shift of all constituents towards the lower molecular weight 28 regions of the chromatogram (higher volume). These experiments emphasise the importance of 29 CL for the higher organisation of these and other factors. 
In order to identify additional interaction partners, we utilised immune-purification and tandem mass tagging mass spectrometry (TMT-MS). In these experiments we immuneprecipitated crude whole membrane extracts $( \pm$ CL) with a monoclonal antibody raised against SecG - a non-essential constituent of the core-translocon SecYEG, and compared native E. coli membranes with those from a strain lacking SecG. Briefly, antibodies were incubated with the extracts which were then mixed with immobilised Protein-G resin. The resin was then stringently washed and the samples were prepared for TMT-MS. A $\Delta \sec G$ strain enabled us to control for non-specific effects of CL (Fig.3a) and for non-specific binding to the antibody and resin (Fig. 3b-d). The three most significant interactors that were retained even without CL were: YajC - a component of the SecDFyajC sub-complex; YfgM - known to interact with SecYEG [22, 23] (Fig. 1h) and YiaD - a suppressor of defective temperature sensitive bamD with an affinity for peptidoglycan (Fig.3b). To the best of our knowledge, YiaD has not previously been identified as a Sec interactor.

The changes resulting from the inclusion of CL were analysed by comparing results obtained 15 from the $\Delta$ SecG and BL21 strains, both in the presence of CL (Fig.3c), and of the BL21 strain in the presence and absence of CL (Fig.3d). As expected, supplementing with CL resulted in 17 more hits. YajC and YiaD were once again identified, suggesting a CL dependent interaction 18 with the secretosome. Inclusion of CL also resulted in identification of SecY, likely due to the core translocon's known dependence on CL for activity and stability. Interestingly, HflC was detected, which is a modulator of FtsH (see also Fig. 1j) and known interactor of YidC [29].

21 Two Sec substrates, OmpA and LptD, which are abundant $\beta$-barrel OMPs, were also detected.

22 Their co-immuno-precipitation with SecG is likely due to capture during transit through SecY.

23 The noted CL enhanced recovery of both OmpA and LptD clients, along with the putative 24 secretosome constituents YajC and YiaD (Fig.3d), is suggestive of a CL-activated and stabilised 25 complex. YajG was also identified when CL was retained in the extracts (Fig.3c,d), which 26 intriguingly is an uncharacterised lipoprotein, and product of the yajG-ampG operon, the latter 27 of which is important for the regulation of PG recycling and remodelling [30-32].

28 There were a few other identified proteins that require explanation. The ribosome subunits 29 are abundant in cells and are known to interact with SecYEG. Pectinesterase was the only hit 30 in the negative control experiment and was ignored as a result. The appearance of subunits of 
1 the ATP synthase is very curious, as a complex with SecYEG was previously identified in

2 mixtures of membrane protein assemblies ejected from native E. coli membranes [33]. Inexplicable hits in proteomic-MS are commonplace. Some may have been identified due to

4 being trapped in the secretosome. False negatives are also a feature as some membrane proteins resist proteolysis and/ or flight for MS. Moreover, extra caution was taken to ensure the protein $\mathrm{G}$ resin was stringently washed, and as a result proteins such as the BAM complex were

7 detected, but not abundant enough to be accurately quantified.

\section{YfgM, PpiD and BAM interact with the HTL}

10 The TMT-MS did not identify an anticipated enrichment of SecDF, BAM or PpiD. So, to

11 verify their membership of the secretosome the SecG co-immunoprecipitants from the extracted 12 native membranes were subjected to Western blotting (Fig. 4a,b). SecD was detected and 13 further enriched by the inclusion of CL, most likely due to HTL stabilisation (Fig. 4a, middle).

14 The same CL enrichment was observed for BamA, supporting previous results that the BAM 15 complex interacts with HTL and not the SecYEG alone (Fig. 4, right) [7, 15]. The specificity 16 of these interactions can be demonstrated through the analysis of membranes prepared from the $17 \Delta \sec G$ strain, from which $\mathrm{SecD}$ and BamA were not co-immunoprecipitated (Fig. 4a, top). 18 Similar experiments also recovered YfgM (highlighted also by TMT-MS; Fig. 3, c) and PpiD 19 (Fig. 4b). In this case, the recovery was marginally increased in the presence of CL (Fig. 4c).

\section{DISCUSSION}

The experiments presented here all point to the existence of a very large assembly spanning the entirety of the Gram-negative cell envelope. The basic structures and function of this supercomplex have already been discussed [15]. Beyond that we identify the involvement of additional components and speculate about the engagement of other factors for the formation of a 'secretosome': a dynamic and versatile hub for envelope biogenesis, quality control and remodelling (Fig. 5).

30 In this scenario, the basic activities of the core translocon SecYEG (Fig. 5a: 1) - protein 31 secretion (Fig. 5a: 2) and membrane protein insertion (Fig. 5a: 3) - are streamlined, adapted or 
1 expanded by the association of various factors as required. The assembly of SecYEG with the 2 ancillary components SecDFyajC and YidC forms the HTL (Fig. 5a: 4), which in all likelihood 3 improves the efficiency of both core activities: YidC for membrane insertion (Fig. 5a: 5) and 4 SecDF for secretion (Fig. 5a: 7) [5, 7, 34, 35]. Interestingly, a dynamic lipid pool at the interface 5 between SecYEG and SecDFyajC-YidC is perfectly positioned to facilitate the former [9].

Chaperones also participate in the late stages of secretion, presumably in order to facilitate successful emergence and onward passage from the Sec-translocon, as well as folding and, 8 where necessary, degradation. PpiD and YfgM, attached to the outer surface of the innermembrane, and those of the periplasm, like Skp and SurA, presumably help facilitate the progression of proteins through and out of the secretosome (Fig. 5a: 8). While more work is

11 needed to unravel the complex and dynamic interplay of the different chaperones at the 12 secretosome, the different chaperones presumably have related, but subtly different activities.

13 Indeed, SurA and Skp are at least partially redundant [10]. Perhaps the individuals, or various

14 combinations of them, are required for different outcomes, such as delivery of $\beta$-barrelled 15 OMPs to the BAM complex at the outer-membrane (Fig. 5a: 9), or early exiting of globular 16 proteins into the periplasm.

17 Translocating proteins must on occasion become misfolded and/ or become trapped within 18 the translocon [36]. This could irreversibly block the secretosome and potentially increase the 19 conductance of small ions, compromising the energy conserving function of the inner 20 membrane. In addition to the loss of functional export sites, this would prove catastrophic if left 21 unresolved. Therefore, the conformational state of such a trapped complex might be recognised 22 by factors, such as the chaperones mentioned above, whose recruitment could facilitate the 23 clearance of denatured protein to nearby or associated proteases in the periplasm (e.g. DegP, 24 FtsH; Fig. 5a: 6). Perhaps the secretosome can even be programmed for retro-translocation of 25 such trapped substrates back through the holo-translocon for cytosolic degradation, for instance 26 by the associated FtsH. Similarly, catastrophic blockages in the secretosome could be resolved 27 by long-range conformational changes back to $\mathrm{FtsH}$, for proteolytic degradation of SecY, and 28 destruction of the defunct assembly. FtsH has indeed been shown previously to act in this way 29 [28].

30 The action of CL on the stabilisation of the holo-translocon [7], the holo-translocon-BAM 31 assembly [15] and the secretosome (shown here) is interesting because the lipid is known to 
1 localise in microdomains [37-40]. Thus, these CL-enriched islands could be sites for

2 localisation of the translocon, by virtue of its affinity for this lipid over any other. These

3 localised hubs could then recruit specific factors of the inner- and outer-membranes to form a

4 bespoke secretosome. This idea resonates with a recently emerging concept of trans-envelope

5 cross-talk for organisation and turnover of the residents of the inner- and outer-membranes [41, 42]. From the point of view of this analogy, it is striking that the outer-membrane islands involved in inter-membrane organisation are indeed enriched in BAM [42].

In this way we propose that specialised secretosomes localise, aided by inter-membrane communication, to form envelope biogenesis hot spots for the provision of the required proteins to the inner-membrane, periplasm and outer-membrane. The high concentrations of CL at these 11 sites, important for secretosome assembly, would also serve to activate SecA - conferring high 12 levels of ATP and PMF driven translocation through the secretosome $[16,17]$. The localisation 13 of CL at areas of high membrane curvature, including division sites and the poles [38, 40, 43], 14 provides focal points for the localisation and activation of trans-envelope secretosome, 15 establishing discrete sites for cell wall biogenesis. On this point, it has been shown that while most SecA is generally membrane associated and mobile, a significant proportion of SecA is

17 immobile ( 25\%) [44]. Perhaps the immobilised SecA is engaged with the secretosome at CL

18 rich envelope biogenesis hubs. Indeed, we know that the affinity of SecA for the holotranslocon is higher where there is an abundance of CL [7]. Moreover, the formation of these static docking sites for SecA are dependent on the PMF [44], which ties in with the conferral of PMF stimulated protein translocation by CL [16], and the requirement of PMF for secretosome activity [15].

The observed broad elution profile of the secretosome constituents we see is presumably 24 due to the existence of various assembled combinations of SecYEG, SecDF-YajC, YidC, BAM and others. This might reflect the situation in native membranes wherein there may be many different requirements at a given time and location. Different super-complexes, with different activities, may be recruited or assembled on site and tuned according to functional requirement, whether that be for delivery of proteins to the periplasm, inner-membrane, outer-membrane and/ or for regions in high demand of quality control. Presumably, the distribution of different

30 complexes in the envelope will change during different stages of the cell cycle, which appears 31 to be the case for the BAM complex [45]. Indeed, The HTL components YidC and SecG along 
1 with BAM were recently shown to localise at sites of cell division, adjacent to divisome proteins

2 where they are presumably recruited to produce the high quantities of proteins required to 3 enable cell division (Fig. 5b) [46]. This also aligns with a proteomics study demonstrating an

4 interaction between SecY and the cell elongation/ division protein RodZ, suggesting the 5 secretosome directly interacts with the divisome [47]. The same study picks up interactions of RodZ and FtsH with BamA. Elsewhere, work in another lab on another organism showed RodZ to interact with SecA and FtsH [48], supporting links between the division and secretion

8 apparatus. Finally, the activity of BamA is supported by an interaction with DolP [49], which

9 in the context of cell division is interesting because DolP is recruited to the mid-cell in the latter

10 stages of this process [50].

11 The active recruitment of different factors to the core Sec-translocon is indeed an interesting 12 concept for both eukaryotic and bacterial secretion systems. The Sec61-complex of the ER does 13 so - forming a holo-translocon with additional components, for functions including membrane 14 protein insertion and glycosylation [51, 52]. In this respect, the association of the Sec62/ Sec63 15 complex plays a role in the recruitment of Hsp70 homologue (BiP) of the ER-lumen to help 16 drive efficient secretion and folding [53]. Interestingly, other more specialist functions have 17 been attributed to the Sec62/ Sec63 pairing [54]. Ancillary components might interact 18 transiently in order to support specific functional requirements, e.g. the ER membrane protein complex (EMC) to facilitate the insertion of more hydrophilic trans-membrane helices [55, 56].

Going back to the core features of the secretosome, i.e. for the delivery and maturation of proteins to the inner membrane, periplasm and outer-membrane, the trans-envelope association

22 of the holo-translocon and BAM complexes brings together a number of interesting motifs, 23 namely the tetratricopeptide repeat (TPR; YfgM, BamD, BepA) and WD40 $\beta$-propeller 24 (BamB). Both motifs provide scaffolds for various other proteins to interact. The same can be 25 said of the inter-membrane associated translocons of the mitochondrial outer- (TOM) and inner26 membranes (TIM23). These TPR scaffolding sites could serve to recruit additional factors (e.g. 27 in bacteria: SurA, Skp or DegP) necessary for transport, such as for retrieval into the periplasm 28 for folding/ degradation, or to facilitate outer-membrane insertion. Perhaps even they could 29 provide staging posts in the thoroughfare for transiting proteins. 
Materials and Methods

$\underline{\text { Plasmids, strains and protein purification }}$

E. coli strains BL21 (DE3), C43 or $\Delta$ secG (KN425 (W3110 M25 $\Delta$ secG::kan)) (a gift from Prof. Frank Duong) were used for all experiments. SecYEG and HTL were expressed and purified as described previously. Briefly, SecYEG was expressed in E. coli C43 from pBAD (Amp ${ }^{\mathrm{r}}$ ), solubilised from the membrane fraction with $1.5 \%(\mathrm{w} / \mathrm{v}) \mathrm{n}$-dodecyl- $\beta$-D-maltopyranoside (DDM, GLYCON Biochemicals GmbSeH) in $20 \mathrm{mM}$ Tris pH 7.5, $130 \mathrm{mM} \mathrm{NaCl,} \mathrm{10 \%} \mathrm{glycerol} \mathrm{(TSG)}$ and purified using nickel-immobilised metal affinity chromatography (IMAC) followed by size

11 exclusion chromatography (SEC) with an appended anion exchange column [20]. The HTL was

12 expressed using the pACEMBL system $\left(\mathrm{Amp}^{\mathrm{r}}, \mathrm{Kan}^{\mathrm{r}}, \mathrm{Cm}^{\mathrm{r}}\right)$ and purified the same as for SecYEG 13 except with $0.002 \%(\mathrm{w} / \mathrm{v})$ cardiolipin supplemented into IMAC and SEC buffers. The plasmid for 14 BAM complex expression, pJH114-BamA-E (Amp ${ }^{r}$ ), was a gift from Prof. Harris Bernstein.

15 Purification of BAM from E. coli $\mathrm{C} 43$ was performed as described previously [15].

17 Production and solubilisation of membranes for co-immunoprecipitations and size exclusion 18 chromatography

Precultures of E. coli BL21 (DE3) containing $100 \mathrm{~mL} 2$ XYT media were inoculated and grown overnight at $37^{\circ} \mathrm{C}$ and $200 \mathrm{RPM}$. The following morning, $10 \mathrm{~mL}$ of preculture was used to inoculate $1 \mathrm{~L}$ of $2 \mathrm{xYT}$ broth in a $2 \mathrm{~L}$ flask and the culture was incubated in the same conditions as described above. When an $\mathrm{OD}_{600 \mathrm{~nm}}$ of 1.0 was achieved, cells were harvested by centrifugation

24 (5000 xg, 10 minutes, $\left.4^{\circ} \mathrm{C}\right)$ and resuspended in $20 \mathrm{~mL}$ of TSG. The sample was then passed through a cell disruptor (Constant Systems Ltd.) for lysis (2 passes at $25 \mathrm{kPSI}$ ). Membranes were clarified from the sample by centrifugation $\left(160000 \mathrm{xg}, 45\right.$ minutes, $\left.4^{\circ} \mathrm{C}\right)$, resuspended to 120

$27 \mathrm{mg} / \mathrm{mL}$ in $\mathrm{TSG}$ and stored at $-80^{\circ} \mathrm{C}$ for future use. Solubilisation was performed by the addition of 28 DDM to $0.5 \%(\mathrm{w} / \mathrm{v})$. After 1 hour of gentle rocking at $4{ }^{\circ} \mathrm{C}$, insoluble material was removed by 29 centrifugation $\left(160000 \mathrm{xg}, 45\right.$ minutes, $\left.4^{\circ} \mathrm{C}\right)$ and the soluble fraction taken for further analysis.

$31 \quad$ Size exclusion chromatography 
2 A Superdex S200 10/300 GL size exclusion chromatography (SEC) column (Cytivia) was 3 equilibrated in TSG buffer supplemented with $0.02 \%(\mathrm{w} / \mathrm{v})$ DDM in the presence or absence of $4 \quad 0.002 \%(\mathrm{w} / \mathrm{v})$ E. coli cardiolipin (Avanti Polar Lipids). With the flow rate set to $0.25 \mathrm{~mL} / \mathrm{min}$, 5 proteins solubilised from $60 \mathrm{mg}$ of wet membrane pellet as described above were injected through 6 a $0.5 \mathrm{~mL}$ loop. $8 \mathrm{~mL}$ after injection, fractions of $250 \mathrm{uL}$ were collected for approximately $10 \mathrm{~mL}$. 7 For SecYEG, HTL and BAM controls, approximately $350 \mu \mathrm{g}$ of purified protein was loaded using 8 the same conditions as for the solubulised membranes. Protein standards were obtained from 9 Merck.

\section{Co-immunoprecipitations}

13 For each reaction, $550 \mu \mathrm{L}$ of solubilised membranes (66 $\mathrm{mg}$ of wet membrane pellet) were

14 prepared as described above and $9 \mu \mathrm{L}$ of purified monoclonal SecG antibody from our laboratory 15 stocks was added. Samples were incubated overnight gently rocking at $4^{\circ} \mathrm{C} .125 \mu \mathrm{L}$ of Protein $\mathrm{G}$ 16 Resin (Amintra) was prepared by washing $250 \mu \mathrm{L}$ of the suspended resin in the manufacturer's 17 storage buffer in a microcentrifuge spin column 3 times with $500 \mu \mathrm{L}$ of buffer containing $250 \mathrm{mM}$ $18 \mathrm{NaCl}, 20 \mathrm{mM}$ HEPES $\mathrm{pH} 8$ (IP buffer) and centrifuge settings of $500 \mathrm{xg}$ for 1 minute at $4^{\circ} \mathrm{C}$. After 19 the final wash, the resin was resuspended in $500 \mu \mathrm{L}$ of IP buffer supplemented with $2 \%$ bovine 20 serum albumin and incubated overnight in the same conditions as the membranes.

The following morning, the resin was washed using the same procedure as described above,

22 but resuspended in $250 \mu \mathrm{L}$ of IP buffer on the final step. $50 \mathrm{uL}$ of material was removed from the

23 solubilised membranes as a loading control and the resin and membranes were mixed and left to 24 gently rock at $21^{\circ} \mathrm{C}$ for 3 hours. The resin was washed in a microcentrifuge spin column 6 times 25 with $400 \mu \mathrm{L}$ IP buffer containing $0.02 \% \mathrm{DDM}$ in the presence or absence of $0.002 \%(\mathrm{w} / \mathrm{v}) \mathrm{E}$. coli 26 cardiolipin. After the $6^{\text {th }}$ spin, the resin was resuspended in $150 \mu \mathrm{L}$ of IP buffer with DDM \pm 27 cardiolipin and $50 \mu \mathrm{L}$ of the suspended resin removed for proteomic analysis. The remaining liquid 28 in the spin column was once again removed by centrifugation and this time collected in a fresh 29 tube for analysis by SDS PAGE (last wash sample). Finally, bound proteins were removed from 30 the resin by addition of $150 \mu \mathrm{L}$ of $1 \mathrm{x}$ LDS sample buffer followed by centrifugation, again 31 collecting the sample in a fresh tube. 
2 SDS PAGE and immunoblotting

4 SEC samples were analysed by SDS PAGE (NuPAGE ${ }^{\mathrm{TM}} 4$ to $12 \%$, Bis-Tris, $1.0 \mathrm{~mm}$, Midi Protein

5 Gel, 26-well) and transferred with a Power Blotter XL System (Invitrogen ${ }^{\mathrm{TM}}$ ) onto $0.45 \mu \mathrm{m}$

6 nitrocellulose blotting paper (Cytivia Amersham ${ }^{\mathrm{TM}}$ Protran $^{\mathrm{TM}}$ ). Immunoblotting was performed

7 by incubating with either purified mouse antibody (SecG, SecY, both diluted 1/10000, from our

8 laboratory stocks) or rabbit antiserum (SecD from our laboratory stocks, or BamA, BamC and

9 BamD, a gift from Harris Bernstein, or a single antisera raised against both YfgM/PpiD, a gift

10 from Prof. Daniel Daley, or FtsH, a gift from Prof. Joen Luirink, all diluted 1/5000), followed by

11 incubation with anti-rabbit or anti-mouse HRP-conjugated secondary antibody (Life

12 Technologies). A homemade ECL kit was used for imaging. Images were acquired for 10 minutes

13 with an Odyssey-Fc imaging system (LI-COR Biosciences) and densitometry performed with the

14 Image Studio Light software (LI-COR Biosciences). Graphs were produced with the Prism 8

15 software package.

17 Tandem mass tag quantitative proteomic analysis of SecG co-IPs

Immuno-isolated samples were reduced $\left(10 \mathrm{mM} \mathrm{TCEP}, 55^{\circ} \mathrm{C}\right.$ for $\left.1 \mathrm{~h}\right)$, alkylated $(18.75 \mathrm{mM}$ iodoacetamide, room temperature for 30 minutes) and then digested from the beads with trypsin (2.5 $\mu \mathrm{g}$ trypsin; $37^{\circ} \mathrm{C}$, overnight). The resulting peptides were then labeled with TMT eleven-plex

22 reagents according to the manufacturer's protocol (Thermo Fisher Scientific, Loughborough,

23 LE11 5RG, UK) and the labelled samples pooled and desalted using a SepPak cartridge according

24 to the manufacturer's instructions (Waters, Milford, Massachusetts, USA). Eluate from the

25 SepPak cartridge was evaporated to dryness and resuspended in 1\% formic acid prior to analysis

26 by nano-LC MSMS using an Ultimate 3000 nano-LC system in line with an Orbitrap Fusion

27 Tribrid mass spectrometer (Thermo Scientific).

28 In brief, peptides in $1 \%(\mathrm{v} / \mathrm{v})$ formic acid were injected onto an Acclaim PepMap C18 29 nano-trap column (Thermo Scientific). After washing with $0.5 \%$ (v/v) acetonitrile $0.1 \%$ (vol/vol)

30 formic acid peptides were resolved on a $250 \mathrm{~mm} \times 75 \mu \mathrm{m}$ Acclaim PepMap C18 reverse phase 31 analytical column (Thermo Scientific) over a 150 min organic gradient, using 7 gradient segments 
1 (1-6\% solvent B over 1 minute, 6-15\% B over 58 minutes, 15-32\% B over 58 minutes, 32-40\% B 2 over 5 minutes, 40-90\% B over 1 minute, held at 90\% B for 6 minutes and then reduced to 1\% 3 B over 1 minute) with a flow rate of $300 \mathrm{~nL} \mathrm{~min}^{-1}$. Solvent A was $0.1 \%$ formic acid and Solvent

4 B was aqueous $80 \%$ acetonitrile in $0.1 \%$ formic acid. Peptides were ionized by nano-electrospray 5 ionization at $2.0 \mathrm{kV}$ using a stainless-steel emitter with an internal diameter of $30 \mu \mathrm{m}$ (Thermo 6 Scientific) and a capillary temperature of $275^{\circ} \mathrm{C}$.

All spectra were acquired using an Orbitrap Fusion Tribrid mass spectrometer controlled 8 by Xcalibur 3.0 software (Thermo Scientific) and operated in data-dependent acquisition mode using an SPS-MS3 workflow. FTMS1 spectra were collected at a resolution of 120 000, with an automatic gain control (AGC) target of 200000 and a max injection time of $50 \mathrm{~ms}$. Precursors

11 were filtered with an intensity threshold of 5000, according to charge state (to include charge states

12 2-7) and with monoisotopic peak determination set to peptide. Previously interrogated precursors

13 were excluded using a dynamic window $(60 \mathrm{~s}+/-10 \mathrm{ppm})$. The MS2 precursors were isolated with

14 a quadrupole isolation window of $1.2 \mathrm{~m} / \mathrm{z}$. ITMS2 spectra were collected with an AGC target of 15 10000, max injection time of $70 \mathrm{~ms}$ and CID collision energy of $35 \%$.

For FTMS3 analysis, the Orbitrap was operated at 50000 resolution with an AGC target

17 of 50000 and a max injection time of $105 \mathrm{~ms}$. Precursors were fragmented by high energy 18 collision dissociation (HCD) at a normalised collision energy of $60 \%$ to ensure maximal TMT 19 reporter ion yield. Synchronous Precursor Selection (SPS) was enabled to include up to 5 MS2 20 fragment ions in the FTMS3 scan.

21 The raw data files were processed and quantified using Proteome Discoverer software v2.1

22 (Thermo Scientific) and searched against the UniProt Escherichia coli (strain B BL21-DE3) 23 database (downloaded January 2020: 4172 entries) using the SEQUEST HT algorithm. Peptide 24 precursor mass tolerance was set at $10 \mathrm{ppm}$, and MS/MS tolerance was set at 0.6 Da. Search criteria 25 included oxidation of methionine $(+15.995 \mathrm{Da})$, acetylation of the protein N-terminus $(+42.011$ $26 \mathrm{Da})$ and Methionine loss plus acetylation of the protein N-terminus (-89.03 Da) as variable 27 modifications and carbamidomethylation of cysteine $(+57.021 \mathrm{Da})$ and the addition of the TMT 28 mass tag $(+229.163 \mathrm{Da})$ to peptide $\mathrm{N}$-termini and lysine as fixed modifications. Searches were 29 performed with full tryptic digestion and a maximum of 2 missed cleavages were allowed. The 30 reverse database search option was enabled and all data was filtered to satisfy false discovery rate 31 (FDR) of 5\%. 
Acknowledgments:

4 We are particularly grateful for the generosity of Dr Harris Bernstein for the kind gifts of the

5 bamABCDE expression construct (pJH114) and antibodies. Thanks to Prof. Daniel Daley for the

$6 \mathrm{PpiD} / Y$ fgM antiserum and to Prof. Joen Luirink the FtsH antibody. Thanks to Kate Heesom of the

7 University of Bristol Proteomics Facility for assistance with proteomics experiments.

9 Funding: This work was funded by the BBSRC (BB/S008349/1 to IC and DWW).

11 Author contribution: DWW and IC conceived and designed experiments; DWW conducted

12 experiments; DWW and IC wrote the manuscript; IC secured funding and led the project.

13

14 Declaration: the authors declare no competing interests.

15

16 Data and materials availability: All data are available in the main text or the supplementary

17 materials. 
bioRxiv preprint doi: https://doi.org/10.1101/2022.01.12.476021; this version posted January 12,2022 . The copyright holder for this preprint (which was not certified by peer review) is the author/funder, who has granted bioRxiv a license to display the preprint in perpetuity. It is made available under aCC-BY-NC-ND 4.0 International license.

2 FIGURES:

a
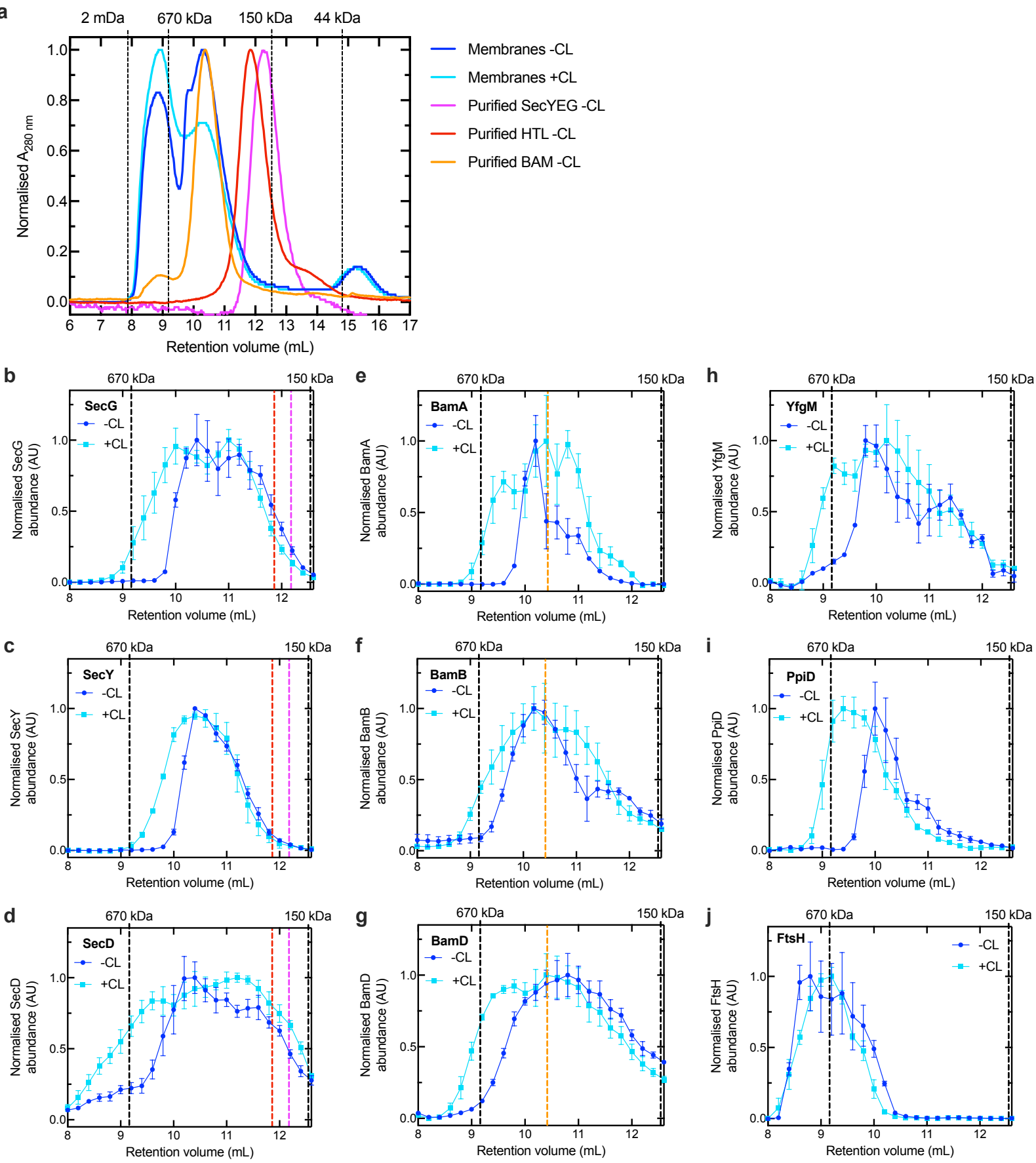
2 Figure 1: Size exclusion chromatography of $E$. coli total membranes.

3 (a) Normalised $\mathrm{A}_{280 \mathrm{~nm}}$ traces of solubilised E. coli total membranes purified on a S200 size 4 exclusion column equilibrated in TSG with $0.02 \%$ DDM and with (+CL) or without (-CL) 5 supplemented cardiolipin as indicated in the legend. Traces of purified SecYEG, BamA-E and 6 HTL controls are also shown. Molecular mass standards were used to calibrate the column 7 (Fig.S1) and their respective masses marked above the graph and indicated with dashed vertical 8 lines.

9 (b-j) Fractions from (a) were immunoblotted for proteins of interest (shown in the top left-hand 10 corner of corresponding graphs). Blotting signal was quantified by densitometry and 11 normalised to the maximum signal value. Both samples were analysed in triplicate 12 (experimental repeats) and errors represent standard deviation. The peak of the elution profiles 13 of the corresponding purified material from (a) are marked by magenta (SecYEG), red (HTL) 14 and orange (BAM) dashed lines. 

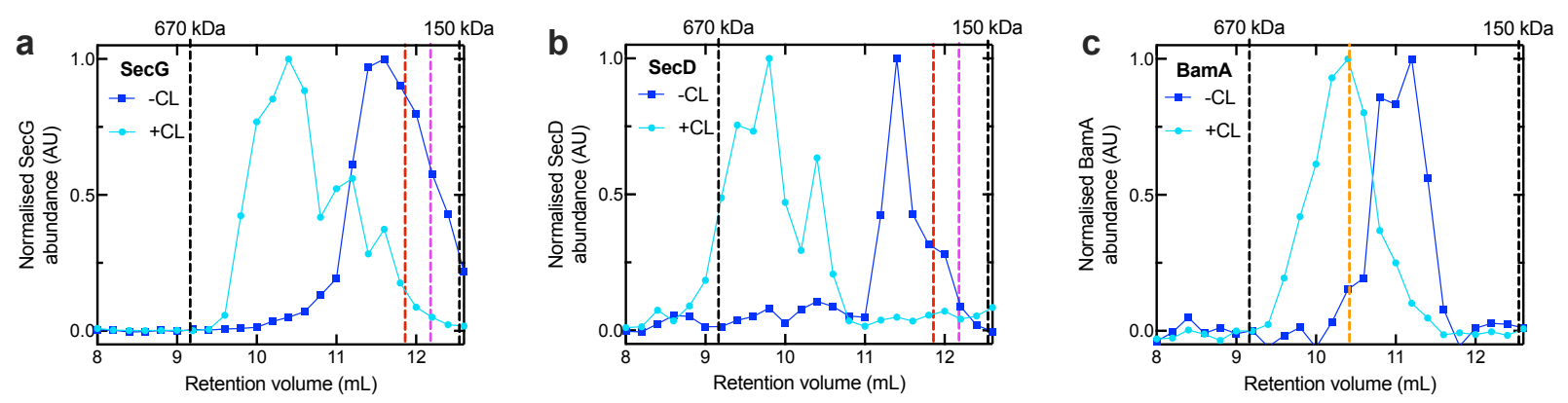

2 Figure 2: Stability of purified membrane complexes.

3 Material collected between 9-10 mL after injection in Fig.1 were pooled and re-loaded onto the

4 same column in TSG buffer with $0.02 \%$ DDM and with (+CL) or lacking (-CL) cardiolipin to

5 analyse secretosome stability.

6

7 

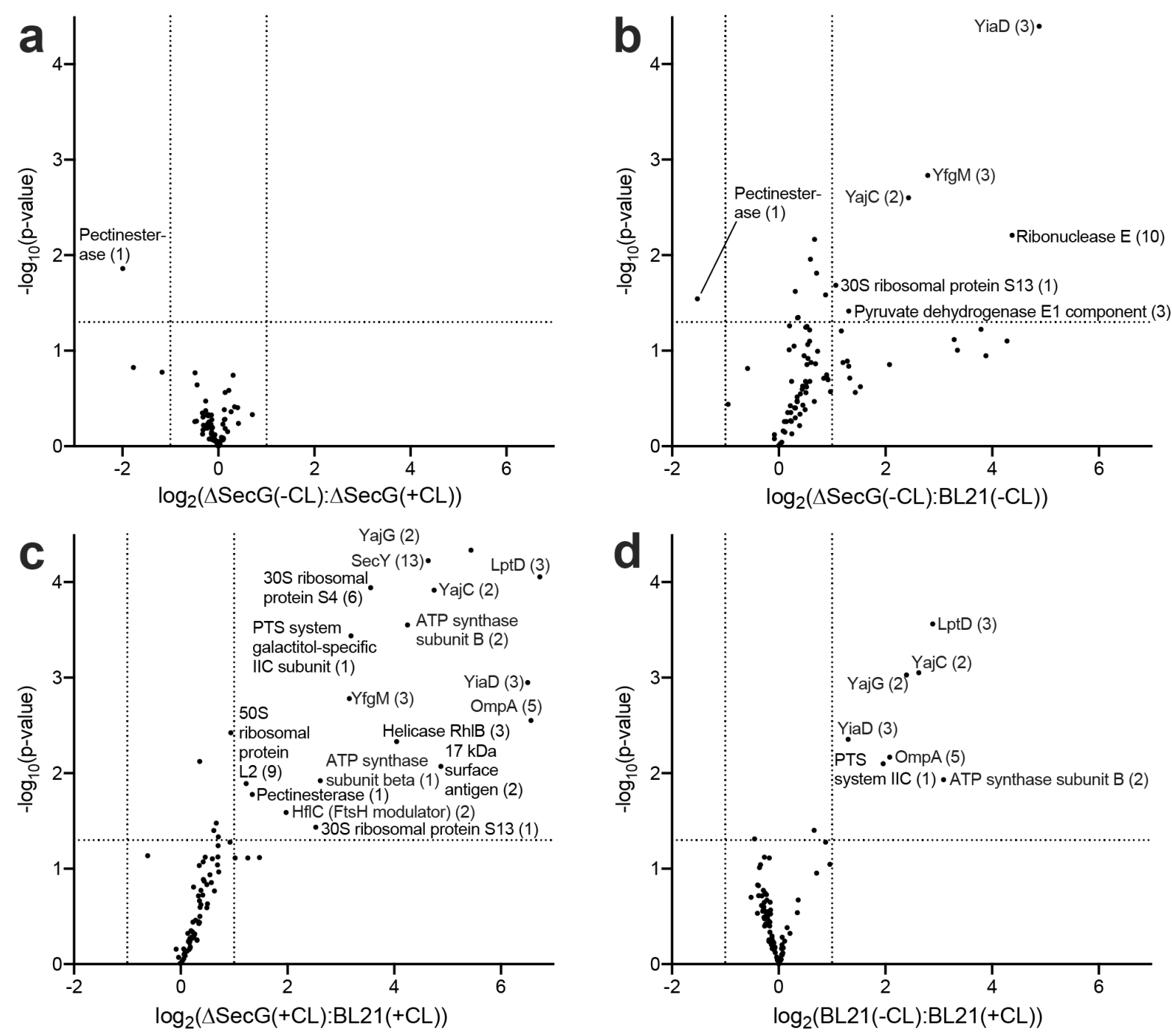

2 Figure 3: Tandem mass tag quantitative proteomic analysis of SecG co-IPs.

3 Samples were prepared from SecG co-IPs of E. coli BL21 (DE3) and E. coli $\Delta$ SecG solubilised

4 membranes in the presence or absence of supplemented cardiolipin, as indicated above respective

5 graphs. $\mathrm{X}$ axis units represent fold change of protein abundance between the two described

6 experiments. Each sample was prepared in triplicate (experimental repeats). An arbitrary cut off is

7 applied at $\log _{2}($ fold change $)=1$ and $p$-value $=0.05$, both indicated by dotted lines for clarity.

8 Proteins with significant abundance changes ( $\mathrm{p}$-value $<0.05$ and $\log _{2}$ (fold change) $>1$ ) are

9 annotated on graphs. 


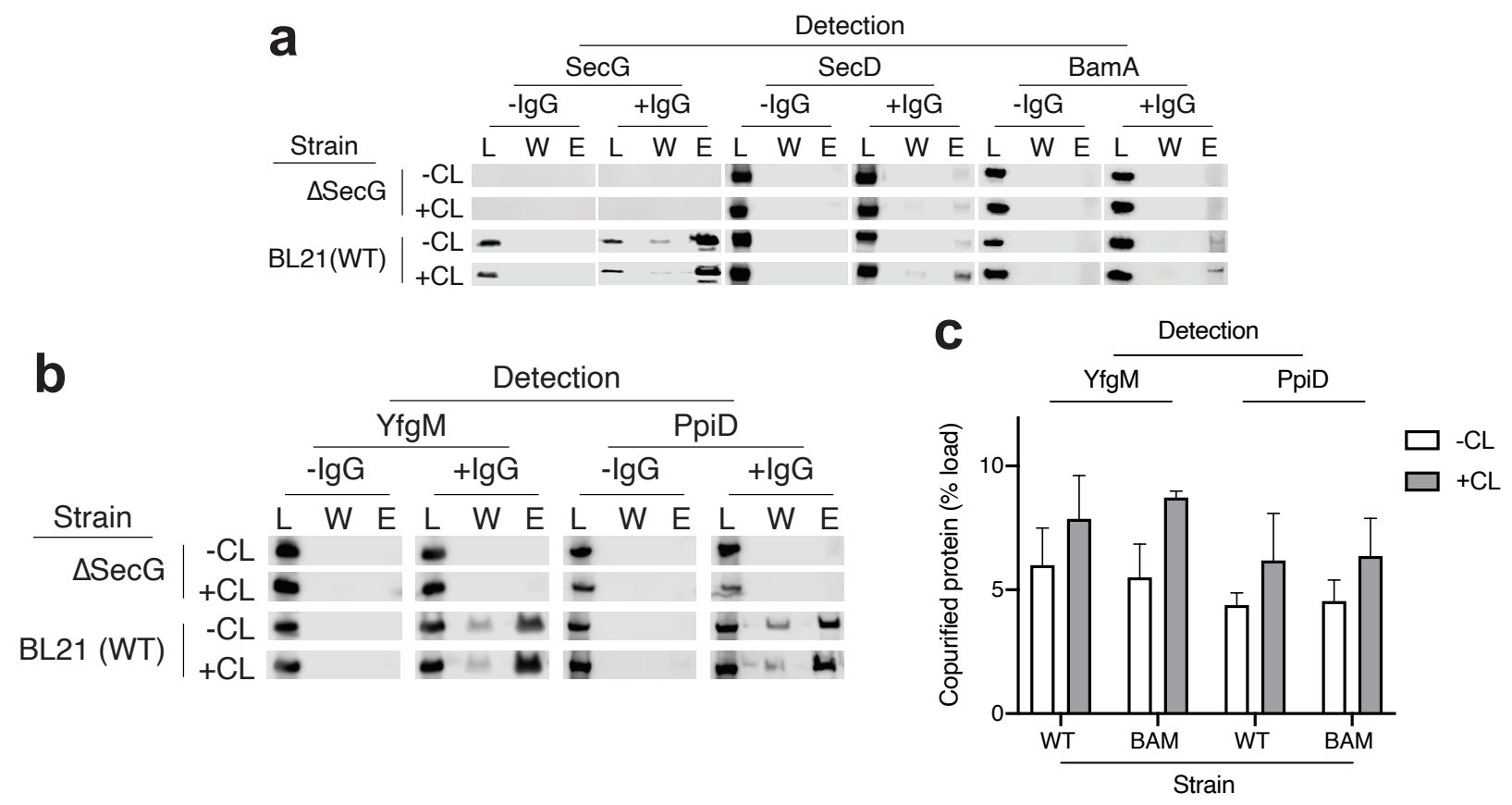

Figure 4: SecG co-immunoprecipitations. Co-IPs were conducted with membranes of E. coli BL21(DE3) or E. coli $\Delta$ SecG solubilised in $0.5 \%$ DDM and washed in the absence (-CL) or presence $(+\mathrm{CL})$ of cardiolipin and in the absence $(-\mathrm{IgG})$ or presence $(+\mathrm{IgG})$ of SecG antibody. In (a) and (b) $\mathrm{L}=$ load (1\% of total material), $\mathrm{W}=$ final wash $(17 \%$ of total material) and $\mathrm{E}=$ eluate (17\% of total material). Immunoblots are shown for SecG, SecD, BamA (a) and YfgM and PpiD 9 standard deviation. 
a

(2)
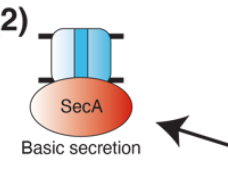

(3)

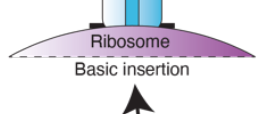

(1)

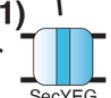

(10) Unkown functions

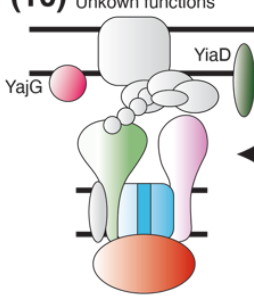

(9)
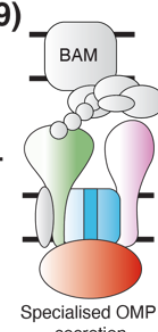

(5)
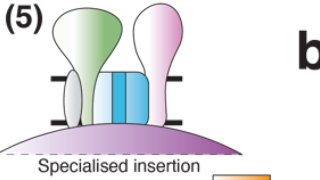

(6)

(4)

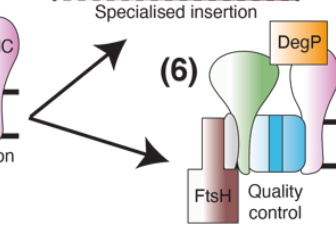

(7)

7) $\downarrow$

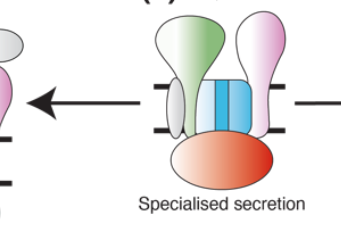

(8)

SurA
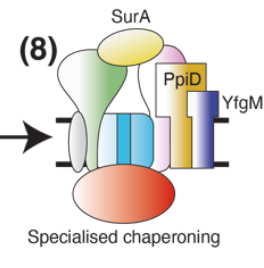

b

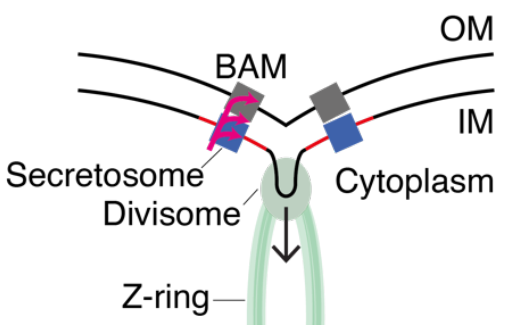

Figure 5: The Secretosome. (a) The core translocon, SecYEG (1), comes in many flavours, some of which are shown here. At the simplest possible level, secretion and inner membrane protein insertion can be conducted by SecYEGA (2) and ribsosome-SecYEG (3) respectively. On formation of the holotranslocon (HTL, 4), the secretosome functions, such as membrane protein insertion (5) and quality control enabled by FtsH (6), can become more specialised. As we have shown in previous work, HTL-SecA can conduct protein secretion (7) and is capable of binding inner membrane (PpiD and YfgM) and periplasmic chaperones (eg. SurA) (8), presumably to prevent aggregation of transiting proteins. HTL also interacts with BAM to facilitate specialised secretion of outer membrane proteins (9), and we have shown in this work that HTL interacts with the lipoproteins YiaD and YajG for unknown functions (10). Note that complexes shown here are not necessarily mutually exclusive. For example, chaperones or quality control proteins may bind

14 localisation and direct interactions of components of the HTL and BAM with components of the 15 divisome, where cardiolipin levels are elevated. 
SUPPLEMENTAL FIGURES:
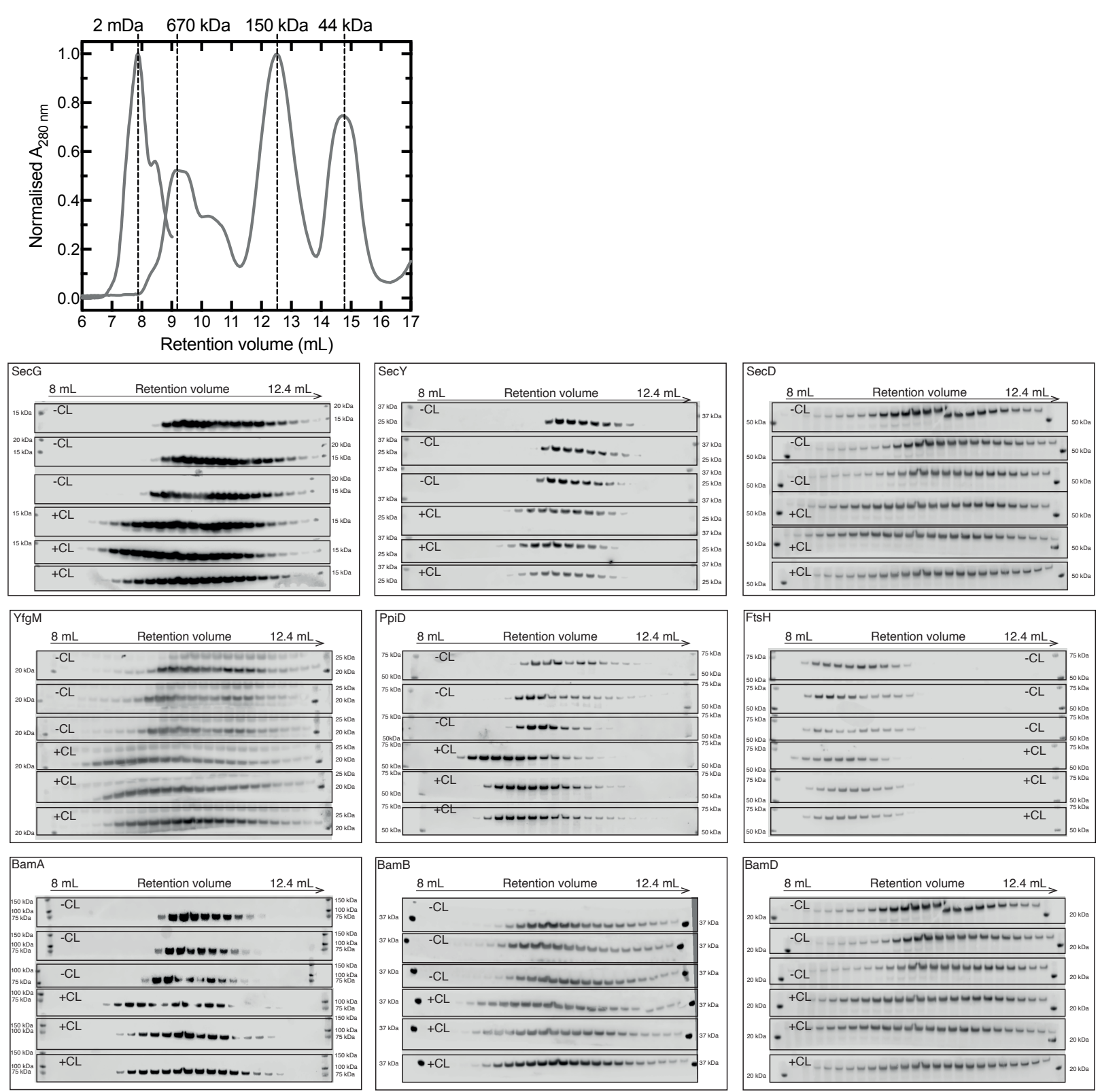

Figure 1 supplement: Immunoblots of S200 fractions of solubilised E. coli BL21 (DE3)

membranes. Molecular mass protein standards (top) were used to calibrate the size exclusion column used in Fig.1. Densitometry was used to calculate relative signal abundance of

6 immunoblots (bottom) and plotted against retention volume in Fig.1b-j. 

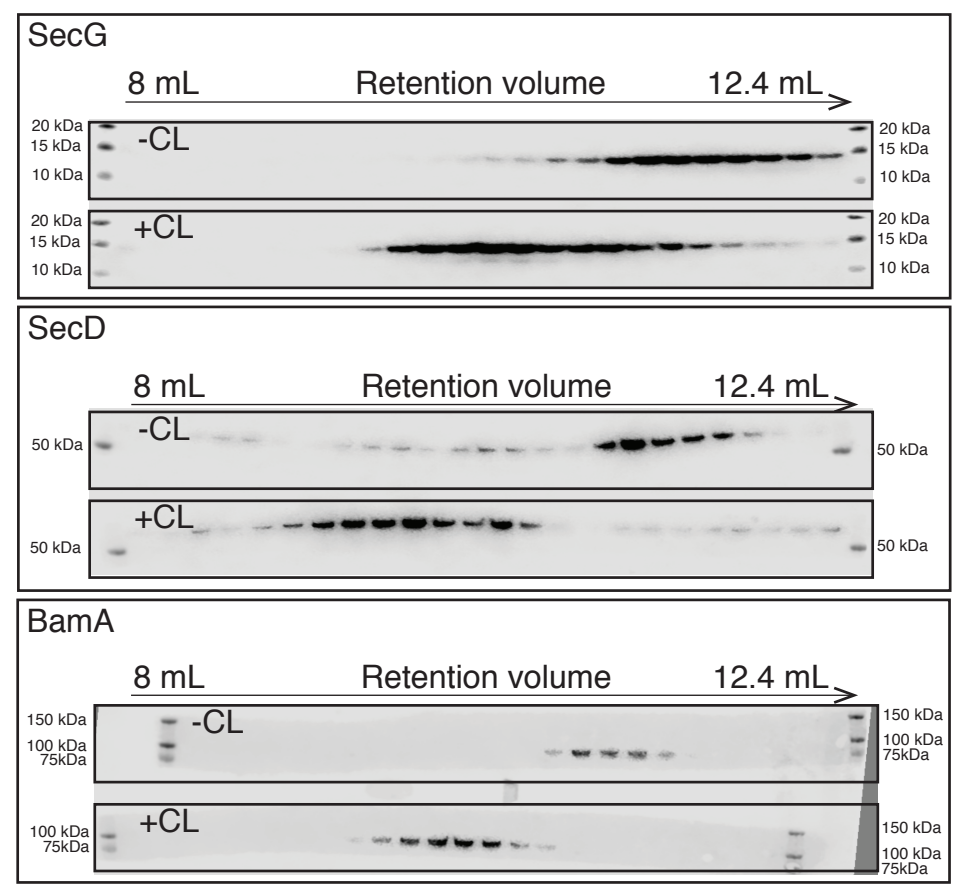

2 Figure 2 supplement: Immunoblots of $\mathbf{S 2 0 0}$ fractions of purified membrane protein

3 complexes. Fractions collected between 9-10 mL after injection in Fig.1 were pooled and

4 reloaded onto the same column in TSG buffer with $0.02 \%$ DDM and with (+CL) or lacking (-

5 CL) cardiolipin to analyse secretosome stability. These raw blots were analysed by

6 densitometry to give relative signal abundances shown in Fig.2. 
a

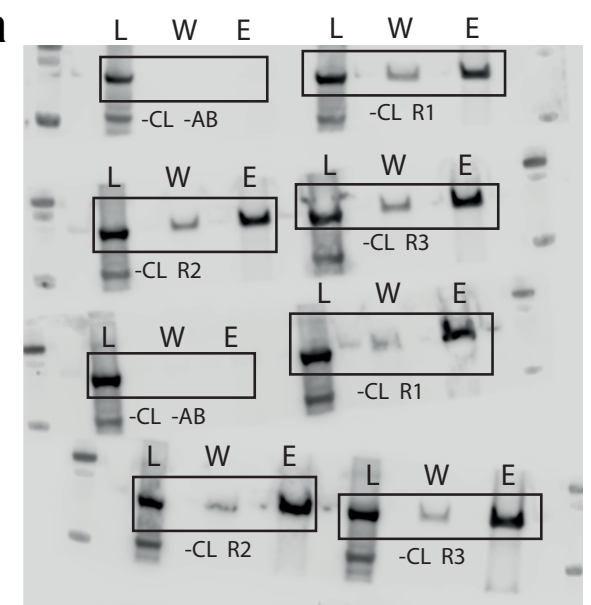

BL21 (DE3): anti-PpiD

b

.

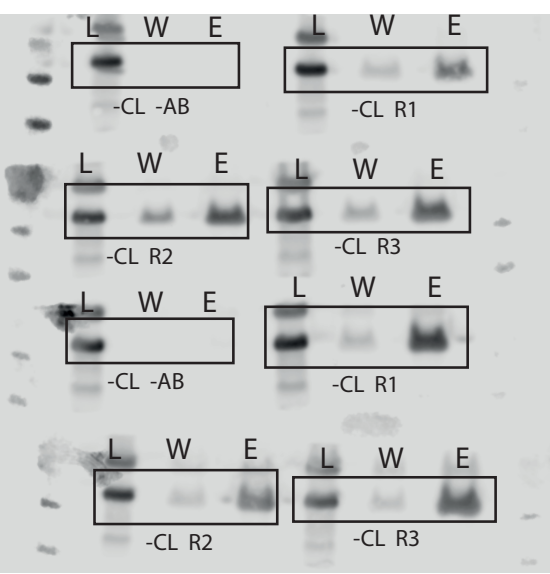

BL21 (DE3): anti-YfgM

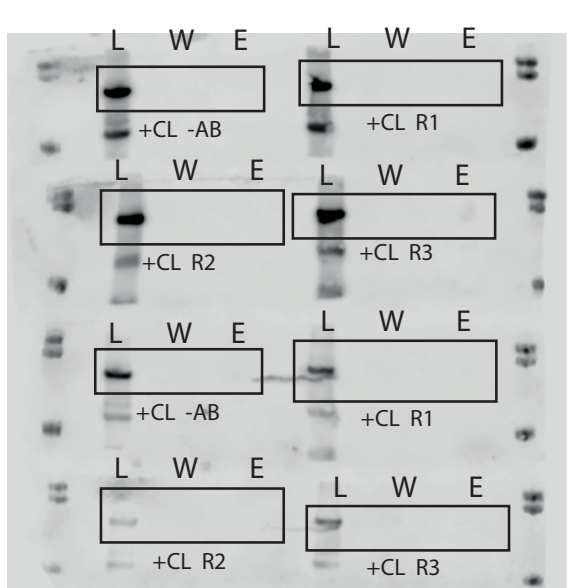

$\Delta$ SecG: anti-PpiD

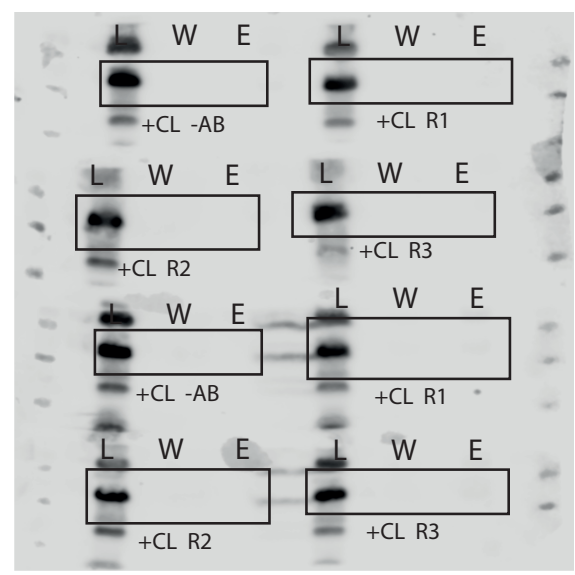

$\Delta$ SecG: anti-YfgM

\section{Figure 4 supplement: PpiD and YfgM raw western blots of SecG co-}

3 immunoprecipitations. Co-IPs were conducted with membranes of E. coli BL21(DE3) (left

4 column) or E. coli $\Delta$ SecG (right column) solubilised in $0.5 \%$ DDM and washed in the absence

5 (-CL) or presence $(+\mathrm{CL})$ of cardiolipin and in the absence $(-\mathrm{AB})$ or presence $(+\mathrm{AB})$ of SecG

6 antibody. $\mathrm{L}=$ load ( $1 \%$ of total material), $\mathrm{W}=$ final wash $(17 \%$ of total material $)$ and $\mathrm{E}=$ eluate

7 (17\% of total material). Samples were prepared in triplicate (experimental repeats, indicated by

8 boxes marked 'R1', 'R2' and 'R3'). 
REFERENCES:

1. Pogliano JA, Beckwith J. SecD and SecF facilitate protein export in Escherichia coli. The EMBO journal 1994;13:554-561.

2. Collinson I. The Dynamic ATP-Driven Mechanism of Bacterial Protein Translocation and the Critical Role of Phospholipids. Frontiers in microbiology 2019;10:1217.

3. Brundage L, Hendrick JP, Schiebel E, Driessen AJ, Wickner W. The purified E. coli integral membrane protein SecY/E is sufficient for reconstitution of SecA-dependent precursor

9 protein translocation. Cell 1990;62:649-657.

4. Cranford-Smith T, Huber D. The way is the goal: how SecA transports proteins across the cytoplasmic membrane in bacteria. FEMS microbiology letters;365. Epub ahead of print June 1, 2018. DOI: 10.1093/femsle/fny093.

5. Samuelson JC, Chen M, Jiang F, Möller I, Wiedmann M, et al. YidC mediates membrane protein insertion in bacteria. Nature 2000;406:637-641.

15 6. Scotti PA, Urbanus ML, Brunner J, Gier JW de, Heijne G von, et al. YidC, the Escherichia coli homologue of mitochondrial Oxa1p, is a component of the Sec translocase. The EMBO journal 2000;19:542-549.

7. Schulze RJ, Komar J, Botte M, Allen WJ, Whitehouse S, et al. Membrane protein insertion and proton-motive-force-dependent secretion through the bacterial holo-translocon SecYEGSecDF-YajC-YidC. Proceedings of the National Academy of Sciences of the United States of

21 America 2014;111:4844-4849.

22 8. Komar J, Alvira S, Schulze RJ, Martin R, Nijeholt JALA, et al. Membrane protein insertion and assembly by the bacterial holo-translocon SecYEG-SecDF-YajC-YidC. The

24 Biochemical journal 2016;473:3341-3354.

25 9. Martin R, Larsen AH, Corey RA, Midtgaard SR, Frielinghaus H, et al. Structure and 26 Dynamics of the Central Lipid Pool and Proteins of the Bacterial Holo-Translocon. Biophysical 27 journal. Epub ahead of print April 9, 2019. DOI: 10.1016/j.bpj.2019.04.002.

28 10. Sklar JG, Wu T, Kahne D, Silhavy TJ. Defining the roles of the periplasmic chaperones 29 SurA, Skp, and DegP in Escherichia coli. Genes \& development 2007;21:2473-2484.

30 11. Rizzitello AE, Harper JR, Silhavy TJ. Genetic evidence for parallel pathways of chaperone 31 activity in the periplasm of Escherichia coli. Journal of bacteriology 2001;183:6794-6800.

32 12. Spiess C, Beil A, Ehrmann M. A Temperature-Dependent Switch from Chaperone to

33 Protease in a Widely Conserved Heat Shock Protein. Cell 1999;97:339-347. 
13. Wu T, Malinverni J, Ruiz N, Kim S, Silhavy TJ, et al. Identification of a multicomponent complex required for outer membrane biogenesis in Escherichia coli. Cell 2005;121:235-245.

14. Ricci DP, Silhavy TJ. Outer Membrane Protein Insertion by the $\beta$-barrel Assembly Machine. EcoSal Plus;8. Epub ahead of print March 2019. DOI: 10.1128/ecosalplus.esp-00352018.

15. Alvira S, Watkins DW, Troman L, Allen WJ, Lorriman JS, et al. Inter-membrane association of the Sec and BAM translocons for bacterial outer-membrane biogenesis. Elife 2020;9:e60669.

16. Corey RA, Pyle E, Allen WJ, Watkins DW, Casiraghi M, et al. Specific cardiolipin-SecY interactions are required for proton-motive force stimulation of protein secretion. Proceedings of the National Academy of Sciences of the United States of America 2018;115:7967-7972.

17. Gold VAM, Robson A, Bao H, Romantsov T, Duong F, et al. The action of cardiolipin on the bacterial translocon. Proceedings of the National Academy of Sciences of the United States of

14 America 2010;107:10044-10049. interactions are required for proton-motive-force stimulation of protein secretion. Biorxiv $2017 ; 202184$.

19. Alvira S, Corey RA, Collinson I, Römisch K. Membrane protein biogenesis by the EMC. Embo J 2021;40:e107407.

20. Collinson I. Projection structure and oligomeric properties of a bacterial core protein translocase. Embo J 2001;20:2462-2471.

21. Bieniossek C, Nie Y, Frey D, Olieric N, Schaffitzel C, et al. Automated unrestricted multigene recombineering for multiprotein complex production. Nature methods 2009;6:447-

22. Jauß B, Petriman NA, Drepper F, Franz L, Sachelaru I, et al. Non-competitive binding of PpiD and YidC to the SecYEG translocon expands the global view on the SecYEG interactome in E. coli. The Journal of biological chemistry. Epub ahead of print November 7, 2019. DOI: 10.1074/jbc.ra119.010686.

29 23. Götzke H, Palombo I, Muheim C, Perrody E, Genevaux P, et al. YfgM is an ancillary 30 subunit of the SecYEG translocon in Escherichia coli. The Journal of biological chemistry $312014 ; 289: 19089-19097$.

32 24. Bittner L-M, Arends J, Narberhaus F. When, how and why? Regulated proteolysis by the 33 essential FtsH protease in Escherichia coli. Biol Chem 2017;398:625-635. 
25. Akiyama Y, Yoshihisa T, Ito K. FtsH, a Membrane-bound ATPase, Forms a Complex in the Cytoplasmic Membrane of Escherichia coli (*). J Biol Chem 1995;270:23485-23490.

26. Akiyama Y, Ehrmann M, Kihara A, Ito K. Polypeptide binding of Escherichia coli FtsH (HflB). Mol Microbiol 1998;28:803-812.

27. Kihara A, Akiyama Y, Ito K. FtsH is required for proteolytic elimination of uncomplexed forms of SecY, an essential protein translocase subunit. Proc National Acad Sci 1995;92:45324536.

8 28. Stelten J van, Silva F, Belin D, Silhavy TJ. Effects of Antibiotics and a Proto-Oncogene

9 Homolog on Destruction of Protein Translocator SecY. Science 2009;325:753-756.

29. Bloois E van, Dekker HL, Fröderberg L, Houben ENG, Urbanus ML, et al. Detection of cross-links between FtsH, YidC, HflK/C suggests a linked role for these proteins in quality control upon insertion of bacterial inner membrane proteins. Febs Lett 2008;582:1419-24.

13 30. Irazoki O, Hernandez SB, Cava F. Peptidoglycan Muropeptides: Release, Perception, and Functions as Signaling Molecules. Front Microbiol 2019;10:500.

15 31. Mallik D, Pal S, Ghosh AS. Involvement of AmpG in mediating a dynamic relationship 16 between serine beta-lactamase induction and biofilm-forming ability of Escherichia coli. Fems

17 Microbiol Lett;365. Epub ahead of print 2018. DOI: 10.1093/femsle/fny065.

18 32. Chahboune A, Decaffmeyer M, Brasseur R, Joris B. Membrane Topology of the

19 Escherichia coli AmpG Permease Required for Recycling of Cell Wall Anhydromuropeptides

20 and AmpC $\beta$-Lactamase Induction. Antimicrob Agents Ch 2005;49:1145-1149.

21 33. Chorev DS, Baker LA, Wu D, Beilsten-Edmands V, Rouse SL, et al. Protein assemblies 22 ejected directly from native membranes yield complexes for mass spectrometry. Science (New 23 York, NY) 2018;362:829-834.

24 34. Gardel C, Benson S, Hunt J, Michaelis S, Beckwith J. secD, a new gene involved in 25 protein export in Escherichia coli. Journal of bacteriology 1987;169:1286-1290.

35. Duong F, Wickner W. Distinct catalytic roles of the SecYE, SecG and SecDFyajC subunits

27 of preprotein translocase holoenzyme. The EMBO journal 1997;16:2756-2768.

28 36. Allen WJ, Watkins DW, Dillingham MS, Collinson I. Refined measurement of SecA29 driven protein secretion reveals that translocation is indirectly coupled to ATP turnover. Proc $30 \quad$ National Acad Sci 2020;117:31808-31816.

31 37. Renner LD, Weibel DB. Cardiolipin microdomains localize to negatively curved regions of 32 Escherichia coli membranes. Proc National Acad Sci 2011;108:6264-6269. 

Bacillus subtilis Marburg Membranes. J Bacteriol 2004;186:1475-1483.

39. Mileykovskaya E, Dowhan W. Visualization of Phospholipid Domains inEscherichia coli by Using the Cardiolipin-Specific Fluorescent Dye 10-N-Nonyl Acridine Orange. J Bacteriol 2000;182:1172-1175.

40. Barák I, Muchová K, Wilkinson AJ, O’Toole PJ, Pavlendová N. Lipid spirals in Bacillus subtilis and their role in cell division. Mol Microbiol 2008;68:1315-1327.

8 41. Rassam P, Long KR, Kaminska R, Williams DJ, Papadakos G, et al. Intermembrane 9 crosstalk drives inner-membrane protein organization in Escherichia coli. Nature

10 Communications 2018;9:1082.

11 42. Rassam P, Copeland NA, Birkholz O, Tóth C, Chavent M, et al. Supramolecular 12 assemblies underpin turnover of outer membrane proteins in bacteria. Nature 2015;523:333-336.

13 43. Oliver PM, Crooks JA, Leidl M, Yoon EJ, Saghatelian A, et al. Localization of Anionic 14 Phospholipids in Escherichia coli Cells. J Bacteriol 2014;196:3386-3398.

15 44. Seinen A-B, Spakman D, Oijen AM van, Driessen AJM. Cellular dynamics of the SecA

16 ATPase at the single molecule level. Sci Rep-uk 2021;11:1433.

17 45. Mamou G, Inns PG, Sun D, Kaminska R, Housden NG, et al. Spatiotemporal

18 organization of BamA governs the pattern of outer membrane protein distribution in growing

19 Escherichia coli cells. Biorxiv 2021;2021.01.27.428258.

20 46. Consoli E, Luirink J, Blaauwen T den. The Escherichia coli Outer Membrane $\beta$-Barrel

21 Assembly Machinery (BAM) Crosstalks with the Divisome. Int J Mol Sci 2021;22:12101.

22 47. Carlson ML, Stacey RG, Young JW, Wason IS, Zhao Z, et al. Profiling the Escherichia

23 coli membrane protein interactome captured in Peptidisc libraries. eLife;8. Epub ahead of print

24 July 31, 2019. DOI: 10.7554/elife.46615.

25 48. Bayramova F, Jacquier N, Greub G. Interactions Screenings Unearth Potential New

26 Divisome Components in the Chlamydia-Related Bacterium, Waddlia chondrophila. Microorg $27 \quad 2019 ; 7: 617$.

28 49. Ranava D, Yang Y, Orenday-Tapia L, Rousset F, Turlan C, et al. Lipoprotein DolP

29 supports proper folding of BamA in the bacterial outer membrane promoting fitness upon

30 envelope stress. Elife 2021;10:e67817.

31 50. Tsang M-J, Yakhnina AA, Bernhardt TG. NlpD links cell wall remodeling and outer 32 membrane invagination during cytokinesis in Escherichia coli. Plos Genet 2017;13:e1006888. 
1 51. Braunger K, Pfeffer S, Shrimal S, Gilmore R, Berninghausen O, et al. Structural basis for

2 coupling protein transport and N-glycosylation at the mammalian endoplasmic reticulum.

3 Science 2018;360:eaar7899.

4 52. Pfeffer S, Pfeffer S, Burbaum L, Burbaum L, Unverdorben P, et al. Structure of the

5 native Sec61 protein-conducting channel. Nature Communications 2015;6:8403.

6 53. Matlack KE, Matlack KE, Misselwitz B, Misselwitz B, Plath K, et al. BiP acts as a

7 molecular ratchet during posttranslational transport of prepro-alpha factor across the ER

8 membrane. Cell 1999;97:553-564.

9 54. Linxweiler M, Schick B, Zimmermann R. Let's talk about Secs: Sec61, Sec62 and Sec63

10 in signal transduction, oncology and personalized medicine. Signal Transduct Target Ther

$112017 ; 2: 17002$.

12 55. Chitwood PJ, Juszkiewicz S, Guna A, Shao S, Hegde RS. EMC Is Required to Initiate

13 Accurate Membrane Protein Topogenesis. Cell 2018;175:1507-1519.e16.

14 56. Shurtleff MJ, Itzhak DN, Hussmann JA, Oakdale NTS, Costa EA, et al. The ER

15 membrane protein complex interacts cotranslationally to enable biogenesis of multipass

16 membrane proteins. Elife 2018;7:e37018. 